Pérez, F. A., Franco, J. C., Vélez, D. A., Vargas, C. M., y Quintero, L. A. (2018). De la responsabilidad social empresarial a la responsabilidad ambiental universitaria. Revista Lebret, 10, 133-155 - ISSN Versión impresa: 2145-5996 • ISSN Versión Web on line: 2357-5468 • https:// doi.org/10.15332/rl.v0i10.2201

\title{
De la responsabilidad social empresarial a la responsabilidad ambiental universitaria ${ }^{1}$
}

\section{From Corporate Social Responsibility to University Environmental Responsibility}

\author{
Fabián Alonso Pérez Ramírez \\ Juan Carlos Franco Montoya ${ }^{3}$ \\ Diego Andrés Vélez Rivera ${ }^{4}$ \\ Carlos Mario Vargas Restrepo 5 \\ Luz Adriana Quintero Osorio
}

\begin{abstract}
Resumen
Este artículo indaga inicialmente por la categoría Responsabilidad Social Universitaria, cuyo primer hallazgo identifica dos grandes tradiciones de su emergencia, una derivada-de, que se fundamenta en tres perspectivas de la Responsabilidad Social Empresarial: teorías de la agencia, teorías de la legitimidad y teorías de la institucionalidad; y la otra tradición es denominada, originaria-en la universidad latinoamericana, que promueve el surgimiento de la Responsabilidad Ambiental Universitaria. A partir de un enfoque cualitativo el diseño documental que permite el abordaje de diversas bases de datos: informes, proyectos y programas ambientales en el interior de un caso de manejo de residuos sólidos en una universidad colombiana. Se puede destacar que en el marco general de las prácticas y acciones ambientales de la universidad se hibridan de forma alternada perspectivas de Responsabilidad Ambiental Universitaria matizadas desde las perspectivas de la Responsabilidad Social Empresarial y la Responsabilidad Social Universitaria.
\end{abstract}

\section{Palabras clave}

Responsabilidad social empresarial, responsabilidad social universitaria, responsabilidad ambiental universitaria.

\section{Códigos de clasificación JEL: M1, M14, Z10}

\begin{abstract}
This article initially investigates the category of University Social Responsibility, whose first finding identifies two great traditions of its emergence, one derived from three perspectives of Corporate Social Responsibility: theories of the agency, theories of legitimacy and theories of the institutionality; and another original tradition-in the Latin American University that promotes the emergence of the University Environmental Responsibility. From a qualitative approach, the documentary design
\end{abstract}

\footnotetext{
Artículo de investigación.

$2 \quad$ Universidad Católica del Oriente. Correo electrónico: fperez@uco.edu.co.

Código ORCID: 0000-0002-4419-0104

3 Universidad Católica del Oriente. Correo electrónico: jfrancodm@gmail.com. Código ORCID: 0000-0001-9357-7244

4 Universidad Católica del Oriente. Correo electrónico: dvelez@uco.edu.co.Código ORCID: 0000-0002-7458-4657

5 Universidad Católica del Oriente. Correo electrónico: cvargas@uco.edu.co. Código ORCID 0000-0002-2490-3808

$6 \quad$ Universidad Católica del Oriente. Correo electrónico: sice.cor@uco.edu.co.
} 
allows the approach of diverse data bases: reports, projects and environmental programs within a solid waste management case in a Colombian university. It is possible to emphasize that in the general framework of the environmental practices of the university perspectives of University Environmental Responsibility are alternately hybridized, nuanced from the perspectives of the Corporate Social Responsibility and the University Social Responsibility.

\section{Keywords}

Corporate social responsibility, university social responsibility, university environmental responsibility.

\section{Introducción}

La Responsabilidad Social Universitaria (RSU) se ha venido convirtiendo en un campo de estudio de alto interés e impacto para las mismas universidades. La tesis que el siguiente artículo se plantea, es que esta responsabilidad no se inscribe en una sola perspectiva, sino que deviene de varias vertientes. La primera que se puede entender como derivada-de las apuestas empresariales, como lo es la Responsabilidad Social Empresarial (RSE), proviene de una RSU desde cuatro dimensiones: la normativa, la mimética, la legítima y la competitiva. Y una segunda RSU originaria-en la propia universidad, que se ha configurado a la luz del interés por comprender los diferentes impactos que sus funciones sustantivas pueden generar en la comunidad y el territorio. Estos impactos se establecen a partir de dos ejes, a saber, el eje organizacional, el cual dinamiza impactos laborales y ambientales, tanto internos como externos. Y el eje académico, que moviliza impactos educativos e impactos de tipo cognitivo epistemológicos e investigativos (Vallaeys, 2014).

Se hace necesario resaltar que, de ambas perspectivas derivada-de y originaria-en, ha venido emergiendo una Responsabilidad Ambiental Universitaria (RAU) que busca trascender las políticas y regulaciones normativas, gestando en la universidad una cultura del cuidado y la sostenibilidad ambiental, haciéndose consciente de que, sus decisiones son generadoras de riesgos e impactos, y que estos tienen afectaciones en la comunidad y en el contexto en general.

\section{Metodología}

El alcance desarrollado en el proceso investigativo es analítico, orientado desde un enfoque cualitativo, donde, a partir del desarrollo de un diseño documental se obtuvo información relacionada con bases de datos e informes expuestos en el Sistema Integrado de Calidad Educativa (SICE) de la Universidad Católica de Oriente (UCO). Para el abordaje del proceso de generación y análisis de información se siguieron los siguientes momentos: ubicación de las fuentes de información, revisión y validación de las fuentes, extracción de la información para la cual se construyó una matriz específica de doble entrada, que permitió el registro y tratamiento de la información, 
la categorización que posibilitó agrupar y desarrollar el análisis cualitativo, a partir de una observación de contenido con alcance interpretativo en un caso en particular de manejo de residuos sólidos en una universidad del país.

Tabla 1. Categorías de análisis y documentos abordados para al proceso investigativo

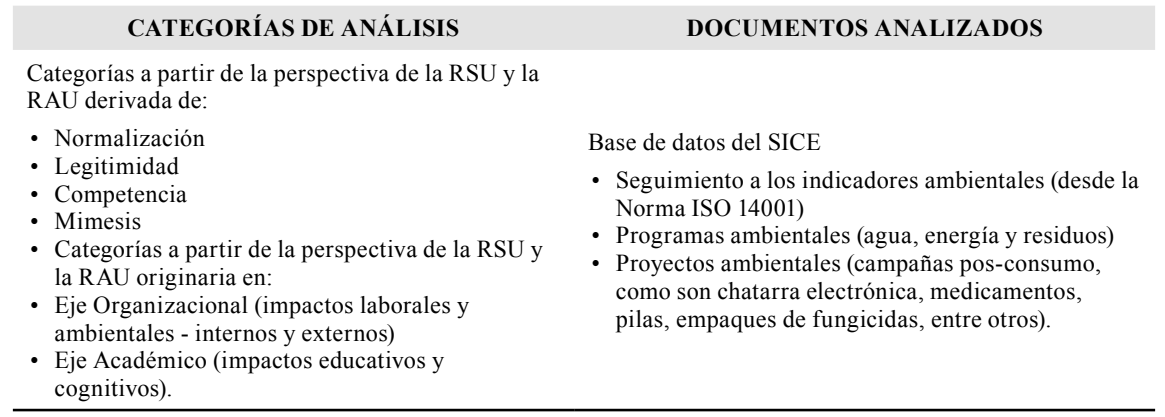

Fuente: Elaboración propia.

\section{Sobre la emergencia de la Responsabilidad Social Universitaria}

La responsabilidad es una cualidad humana inmemorial que se manifiesta como actitud, por ende, es una construcción de sí mismo en relación con los otros en diversos ambientes naturales y sociales; es una respuesta en tanto implica hacerse cargo de las actuaciones humanas y de los efectos e impactos que cada uno trae consigo. "Lo anterior significa que la responsabilidad y la subsecuente imputabilidad ética no tienen sentido, no podrían explicarse, si el hombre no puede pensarse como libre" (López, 2018, p. 12).

Es de anotar, que la responsabilidad ha sido objeto de estudio de la filosofía en el interior de la teoría axiológica, al igual que en el ámbito de la ética, los estudios jurídicos, y en general de la deontología en todas las profesiones. Más recientemente aparece en el ámbito de los estudios organizacionales de la educación y de la hoy denominada universitología, de ahí que, exista una larga tradición de pensadores y escuelas que han hecho de esta actitud humana una categoría de reflexión que permanentemente ha generado acumulados conceptuales, teóricos, legales y prácticos.

A continuación, se aborda a manera de aproximación al estado de la cuestión, el concepto de RSU no sin antes plantear inicialmente que, a partir de la literatura revisada se nombran dos grandes tradiciones para el abordaje de la emergencia de este concepto, la primera la llamaremos derivada-de y, la segunda, originaria-en. 


\section{La Responsabilidad Social Universitaria derivada-de la Responsabilidad Social Empresarial}

La primera forma, derivada-de, determina el surgimiento de la RSU y de la RSE o corporativa, es decir, emana de las teorías de la administración, aquí, Camacho, Fernández y Miralles (2005), y Gaete (2015) coinciden en que, dicha influencia se presenta desde las primeras concepciones de la RSE en tanto filantropía empresarial, pues se espera que las acciones empresariales den respuesta a las expectativas de la sociedad, partiendo del principio de buenos ciudadanos corporativos (Carroll, 1991). De otro lado, Jones (1995) la define como la noción de que las empresas tienen una obligación con sus stakeholder ${ }^{7}$, trascendiendo los intereses de los accionistas, y más allá de lo prescrito por ley o contrato sindical, lo que indica una participación que va más allá de la mera propiedad.

Así mismo, y desde las teorías del stakeholders (Freeman, 1984; Fassin y Gosselin, 2011; y Scholl, 2001), se promueve la satisfacción de expectativas de los grupos de interés, no solo con visión filantropía de la gestión organizacional, sino también, como parte de las decisiones estratégicas de los directivos, pues, se debe actuar de acuerdo a las necesidades de los involucrados (Rasche \& Esser, 2006). Es necesario entonces partir de preguntas como ¿Cuáles son los grupos de interés que merecen o requieren atención de la empresa? y ¿cuáles no? tomando como punto de inicio el nivel de poder, legitimidad y urgencia de cada uno de los involucrados en la dinámica empresarial (Mitchell, Agle, \& Wood, 1997).

Adicional a lo anterior, resulta conveniente discutir sobre el nivel de competitividad percibido por las organizaciones en relación con las prácticas de RSE o RSU, entendiendo la competitividad como las fortalezas de una organización, en comparación con las otras (Murths \& Lenway, 1998). Es importante abordar este término, pues algunos estudios han comprobado que existe una relación positiva entre las RSE o RSU y los futuros resultados financieros (Griffin y Mahon, 1997; McWilliams y Siegel, 2001, citados en Vilanova, Lozano y Arenas, 2009), este enfoque, puede ir en contravía con la visión de responsabilidad filantrópica presentada por Carroll (1991) o por lo que se espera de una auténtica RSU, sin embargo, la visión de Freeman (1984) sostiene que el resultado de obtener una ventaja competitiva sostenible y generadora de valor económico, resulta de la retribución emocional entregada por la comunidad y por la inversion que hizo la empresa en crear valor para las partes involucradas. Lo anterior se dinamiza y se fortalece tanto en el corto como el largo plazo, mediante la adopcion de prácticas de RSE o universitaria. En el fondo, al parecer existe una relacion directa entre la competitividad y la creacion de valor, no obstante, debe hacerse claridad en que aún hay un alto grado de subjetividad en esta relacion, motivo por el cual la responsabilidad social aprovecha estos vacios para redefinirse

$7 \quad$ Según Evan \& Freeman (1988) entenderemos por Stakeholder a aquellos beneficiados de, o perjudicados por el actuar natural de las organizaciones, y cuyos derechos son violados o respetados por las mismas. 
constantemente, y evolucionar o permear otras organizaciones. En contraste con lo anterior, se empiezan a crear definiciones como la aquí planteada (RSU) y otra como la Responsabilidad Ambiental (Trujillo y Vélez, 2006) que al entenderse como prácticas voluntarias, se espera o se tiene la expectativa de que las relaciones que surgen derivadas de esta práctica cumplan los compromisos pactados, sin la necesidad de que exista una sanción formal (Ángel, 2008).

Ahora bien, en la pretensión de trasladar la perspectiva económica de maximización de la competitividad y de los beneficios empresariales a los bienes públicos, dentro de ellos la educacion, se genera un conflicto dada la imposibilidad de reducirlos a esta simple perspectiva económica que no asume las dimensiones pública y social, y que por el contrario demandan su indivisibilidad, su irrenunciabilidad y su orientacion hacia el interes público.

En tal sentido, la teoría de la agencia, trabajada por Ross (1973), Jensen y Meckling (1976) y Bohren (1998), promueve el surgimiento de una RSU, derivada del conflicto enunciado anteriormente, y que se plasma en una contraposición entre los propósitos de la empresa (pública o privada) y los de la universidad que promueven una responsabilidad social sustentada en un modelo de autonomía y deferencia de la gobernanza empresarial tradicional (Santos, Núñez y Alonso, 2007) y en la trasparencia informativa, social, económica y ambiental, lo cual ratifica el tratamiento diferenciado y direccionado que debe darse a la educación.

Es así como se posibilitaría corregir de intereses entre los ejercidos por organizaciones con ánimo de lucro y los de las universidades que, a partir de la profesionalización de la gestión universitaria suscitaría la reestructuración de los sistemas de gobernanza con la intención de generar mayor autonomía, más allá de una rendición de cuentas centrada en los formatos de auditoría y control de la calidad, que pone énfasis en la obtención de los objetivos trazados.

La RSU, entonces, ponen el acento en los modelos de gestión socialmente responsables, que acogen las necesidades y expectativas de los diversos grupos que de una u otra forma están siendo afectados por la existencia y acción de las universidades, promoviendo una nueva gobernanza (Melle, 2007). Así, la RSU se asume desde la colaboración de las comunidades locales y regionales, como también, del personal de la universidad misma y de sus estudiantes. La universidad desde este enfoque se entiende como un organismo líder en la creación de una sociedad sostenible a través de una amplia gama de participación de los stakeholders (Cortese, 2003; Galán, 2006), de ahí, que sea la sociedad la que delimita las responsabilidades de la universidad y que, a mayor autonomía de las universidades, mayor grado de integración de esta a la sociedad.

Por su parte, desde las teorías de la legitimidad (Suchman, 1995; Gray, Owens, Adams, 1996; Reverte, 2009), la RSU es comprendida como contrato social entre las universidades y la sociedad para la mejora de su imagen y su reputación social, 
entendida la legitimidad como la percepción generalizada de que las acciones de una organización son deseables y adecuadas según un sistema de valores, creencias, normas y definiciones construidas social e históricamente (Suchman, 1995). Es así como la RSU en esta perspectiva, se entiende como el cumplimiento de un contrato social que satisface una serie de demandas a cambio de la aprobación de su accionar, lo que además implica la readaptación del contrato social según demandas en cada tiempo.

Desde la teoría institucional (Vaca, Moreno y Riquel, 2007, Lapsley, 1999, Ashworth, Boyne y Delbridge, 2007) se plantea una tendencia isomorfa en las instituciones (incluida la universidad), donde sus cambios responden a tres tipos de mecanismos: de coerción (según marcos legales), de mimetismo (imitación de prácticas) y de normalización (estandarización de procesos según profesionalización de actividades) (Powell y Di Maggio, 1999). Se presentan otras formas de asumir la RSU, por ejemplo, en tanto corresponsabilidad institucional entre las administraciones educativas de un territorio, por un lado, y las universidades, por el otro, lo que ha sido objeto de críticas en la presentación de la RSU como un modelo alternativo de gestión que devela como fundamento una presión ejercida por diferentes organismos internacionales (De la Torre, 2009), promoviendo en las universidades el enfoque de RSU como política de responsabilidad social, como lineamiento institucional que responde a la tendencia isomorfa, ya sea de coerción legal, imitación de prácticas de otras universidades o incluso como normalización y estandarización de sus procesos.

Por último, la RSU se entiende como el cúmulo de estrategias derivadas de la teoría de los recursos y capacidades (Porter y Kramer, 2011, Sousa, Outtes, Pasa \& Farache, 2010 y Grant, 2006), es decir, la RSU se comprende desde esta perspectiva como un mecanismo de competitividad y diferenciación entre las universidades, como un elemento de rentabilidad, éxito y supervivencia de la organización (Porter y Kramer, 2006), siendo la RSU un mecanismo de competitividad en el desarrollo de herramientas en la planificación estratégica y de gestión de las universidades (Santos et al. 2007).

Todas estas perspectivas de comprensión de las teorías de la RSE se pueden desplegar en una forma de concebir la RSU en tanto una estrategia de gestión que intenta integrar esfuerzos, maximizando los recursos, capacidades y beneficios en virtud de la razón de ser de la universidad, entendida esta como organización capaz de articular voluntariamente compromisos y acciones en el uso sistemático de sus funciones, promoviendo el respeto a todos los participantes en el crecimiento social y universitario, junto al cuidado del ambiente para la sostenibilidad de las organizaciones, entre ellas las universidades.

No obstante, no basta asociar la comprensión de la RSU a las asunciones de cada teoría organizacional frente a la RSE, se sugiere más bien, pensar si es posible otra perspectiva de comprensión en la emergencia de la RSU en el seno mismo de la universidad, entendida esta última como institución social, portadora y generadora de saber en relación directa con los patrimonios culturales, científicos, técnicos, 
tecnológicos y demás acerbos de la humanidad. Así, autores como Vallaeys (2014) plantean la RSU como una política de gestión universitaria que se va desarrollando en Latinoamérica, que recoge elementos de la extensión universitaria y de la democratización de la universidad impulsada tras el Movimiento de Córdova en 1918 y que hundiría sus raíces en el Primer Congreso Internacional de Estudiantes Americanos de 1908 en Uruguay, donde se plantea que en la razón de ser de la universidad latinoamericana está su función social y de movilización de la sociedad. Veamos entonces, en qué consiste la RSU originaria-en la universidad.

\section{La Responsabilidad Social Universitaria originaria-en la universidad latinoamericana}

La segunda forma de abordar la emergencia de la RSU la denominamos originaria-en, la cual plantea que su aparición se da en tanto función social de las universidades (Bustos e Inciarte, 2012 y Vallaeys, 2014). Desde esta perspectiva, la RSU hunde sus raíces en lo que se ha denominado el modelo de universidad latinoamericano, en su apuesta de cara a los problemas sociales expresada a través del Congreso Internacional de Estudiantes Americanos llevado a cabo en 1908 en Uruguay y con mayor fuerza en el Manifiesto de Córdoba que "reclama un gobierno estrictamente democrático y sostiene que el demos-universitario, la soberanía y el derecho a darse el gobierno propio" (Manifiesto de Córdoba, 1918, p. 1) implicaría el ejercicio consciente de las diversas problemáticas del entorno para trascender la visión profesionalizante de la universidad, heredada del modelo Napoleónico (Jiménez y Pérez, 2017). En esta misma línea, hacia 1949, se reorienta el espíritu de la universidad más allá del ejercicio de la docencia y la investigación en el marco del primer Congreso de Universidades Latinoamericanas (Ojeda y Álvarez, 2015) que redefine la extensión universitaria

Por su contenido y procedimiento, la extensión universitaria se funda en el conjunto de estudios y actividades filosóficas, científicas, artísticas y técnicas mediante las cuales se auscultan, exploran y recogen del medio social, nacional y universal, los problemas, los datos y los valores culturales que existen en todos los grupos sociales (Ojeda y Álvarez, 2015, p. 1).

Esta forma de comprender la extensión universitaria, implica por lo menos dos asuntos fundamentales, en tanto fundacional de la RSU en clave de función social de la universidad. Por un lado, su sentido fundacional asociado a la calidad de estudios que reflexionan de cara al ámbito social reconociendo sus problemáticas, más allá de los problemas disciplinares; y por otro, el sentido de acogida de diversos grupos sociales y sus valores, como posibilidad de exploración y generación de conocimiento desde diversas perspectivas disciplinares. Así, hacia 1972 y en el marco de la Segunda Conferencia Latinoamericana de Difusión Cultural y Extensión Universitaria se plantea: 
Una universidad abierta al diálogo, consciente de los procesos de comunicación con la sociedad, una universidad capaz de comprender los problemas que surgen en la sociedad e incluso, los que ella misma pone y poder dar solución a ellos con las comunidades que hacen parte de ella (Jiménez, Pérez, 2017, p. 131).

Esta universidad, como bien lo indica Jiménez y Pérez (2017) se convierte entonces en un escenario abierto, que va más allá de la RSU derivada de la RSE, como antes se mencionó. En este sentido, Vallaeys (2014) plantea la posibilidad de un nuevo modelo que asuma la RSU, trascendiendo la visión mercantilista de la gestión universitaria, aduciendo que la RSU se puede convertir en una nueva política que atienda no solo las problemáticas organizacionales, académicas e investigativas de la universidad, sino también, las ambientales.

Es aquí donde se puede identificar una perspectiva de la RSU que nace o es originaría en la misma universidad, ya que esta se diferencia según Vallaeys (2014) de "la tradicional extensión solidaria como un mero compromiso unilateral declarativo y obliga a cada universidad a poner en tela de juicio sus presupuestos epistémicos y su currículo oculto" (p. 105). Si se observa con detalle, esta perspectiva obliga a la universidad, como lo plantea Franco (2018) a una "revisión, autoobservación y crítica permanente respecto a la pertinencia, coherencia y legitimidad de sus procesos" ( $\mathrm{p}$. 146). Dichos procesos relacionados con cada una de las funciones que sustentan la universidad, desde la docencia, la investigación y la extensión, pero que trascienden a los mismos efectos e impactos de estas en el ambiente.

\section{Sobre la Responsabilidad Ambiental Universitaria}

No es posible pensar una RSU al margen de una RAU, ya que, la primera según Franco (2018) "obliga a las universidades a pensar como organizaciones" (p. 146), la cual, en palabras de Vallaeys (2014), la denomina como la gestión de los impactos generados por las universidades, vistas desde una perspectiva sostenible y justa, está como parte esencial de las mismas funciones.

Teniendo presente lo antes mencionado, Vallaeys (2014) plantea que, "la RSU no es un compromiso ético unilateral, es respuesta obligada a deberes sociales y medioambientales mediante el tratamiento de los propios impactos negativos de la universidad" (p. 107). De esta manera, el autor señala cuatro tipos de impacto, relacionados con lo expuesto anteriormente, los que la universidad debe gestionar en forma socialmente responsable, estos tipos se dividen en dos ejes: i) eje de impactos organizacionales: laborales y ambientales internos que tienen que ver con las dinámicas propias de las funciones de la universidad y externos que hacen referencia a los impactos sociales ambientales que afectan a todos los agentes externos con los cuales se encuentra relacionada la universidad y ii) eje de impactos académicos: educativos que refiere a aquellos impactos formativos hacia los estudiantes y cognitivos y epistemológicos, que tiene que ver con "la producción de conocimientos científicos 
y el rol político de la ciencia" (Vallaeys, 2014, p. 111). Se puede afirmar entonces que, no es posible ver la RSU, separada de la responsabilidad ambiental que en la actualidad tiene la universidad, lo que le exige a esta establecer protocolos claros de organización para la toma de decisiones en relación con lo socioambiental. Una reflexión teórica clara debe contener y reconocer la relación inseparable de estas dos categorías de responsabilidad.

La figura 1 intenta mostrar que los impactos de la universidad son más complejos y dinámicos de lo que se ha venido estableciendo e implementando como RSE del cual deriva la RSU, implementados desde marcos operacionales como las normas ISO 14001 (2015) que abala y ofrece directrices al tema de gestión ambiental en las organizaciones y la norma ISO 26000, que plantea directrices para el cumplimiento de la responsabilidad social de las organizaciones, teniendo presente los efectos de estas en lo social y ambiental. Vallaeys (2014) expresa que dicho modelo amplia la perspectiva hegemónica de campus sostenible, propuesta por las visiones de América del Norte y Europa, al afirmar que "la concepción latinoamericana de la RSU es más radical que la del Norte, porque se apoya en la tradición latinoamericana de la misión social universitaria que las universidades del Norte han descuidado ampliamente" (p. 108).

Figura 1. Gestión justa y sostenible de los impactos universitarios

\section{Es la gestión justa y sostenible de los impactos universitarios}
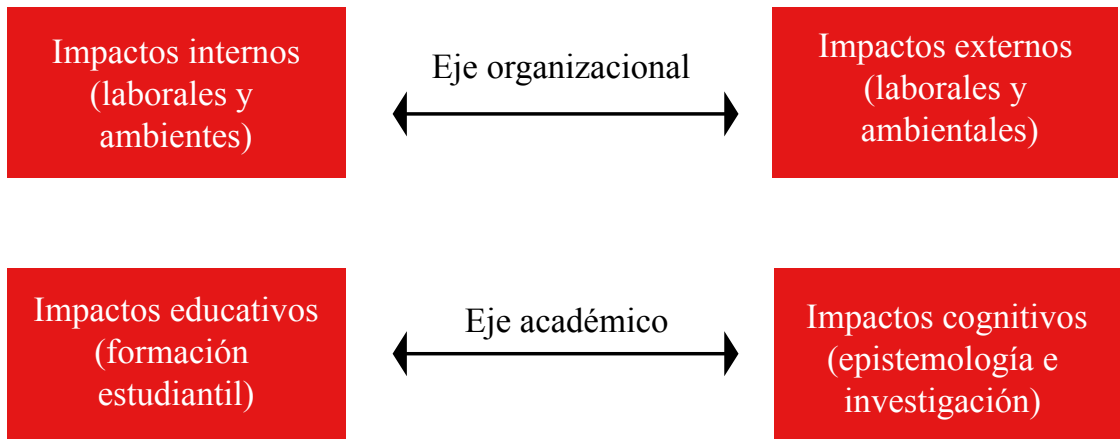

Fuente. Responsabilidad Social Universitaria: un nuevo modelo de universidad contra la mercantilización (Vallaeys, 2014).

Ahora bien, algunas ventajas de este modelo que se pueden destacar, según los planteamientos de propio Vallaeys (2014), son: 
- Permite reconocer la evolución que ha tenido el concepto de RSU, teniendo presente que no es una mera aplicación de normatividades previamente establecidas, sino más bien que invita a las universidades a la realización de un acto consciente en relación con la identificación, análisis de sus propios impactos de forma genuina, los cuales pueden ser atendidos desde las propias capacidades y competencias académicas de la universidad.

- La posibilidad de entender la complejidad, profundidad y amplitud de la RSU y la necesidad de configurar una perspectiva propia en cada universidad.

- Permite ampliar la visión de una RSU a una RAU, desde la cual se pueda "desarrollar una crítica integradora frente a la estrechez del paradigma latinoamericano de la extensión, que tiende a reducir la responsabilidad social de la universidad al mero compromiso solidario con poblaciones necesitadas" (Vallaeys, 2014, p. 108).

Desde lo anterior, es posible afirmar que el enfoque conceptual que mejor configura la comprensión y el significado relacionado con la perspectiva de la RAU, tiene que ver con entender la universidad como una organización con responsabilidad social y ambiental, que busca desde la docencia, la investigación, la extensión, proyección social y la gestión interna identificar y atender los propios impactos que esta genera en las personas, los grupos, organizaciones y el territorio en general, teniendo en cuenta los impactos cognitivos, educativos, sociales y ambientales que su misma actividad genera.

Esta forma de comprender la responsabilidad ambiental universitaria, se puede establecer por lo menos en dos perspectivas, la primera en clave de función social de la universidad, que tiene que ver con todos aquellos factores implicados en los procesos de comunicación e interacción con la misma sociedad, que hacen referencia a la capacidad de entender los diversos problemas que surgen en el marco de la relación universidad-sociedad, y que emergen de la puesta en marcha de sus funciones sustantivas, dando paso, a partir de dicha relación, a proponer soluciones desde y con las comunidades que hacen parte de ella, como lo expresan Jiménez y Pérez (2017).

La segunda en clave ambiental, desde la cual se hace necesario reconocer los efectos e impactos que los desarrollos de las funciones sustantivas generan en el interior de la universidad y en la relación con el territorio, vistos desde una mirada integral, asumiendo como líneas fuerza: la decisión, el riesgo, el impacto y la intervención; y como categorías base: lo físico-material, lo subjetivo-psíquico, lo intersubjetivo-interaccional y lo social-contextual.

Como se viene afirmando, la universidad como organización involucra un conjunto de funciones sustantivas a partir de las cuales, toman decisiones y actúan generando profundos impactos en el acontecer educativo, económico, social y ambiental en el que se desenvuelve. Dichas funciones se configuran como campos interrela- 
cionales e interactuantes, es decir, como una red de relaciones que la universidad dinamiza. En este accionar dinámico participan los diferentes agentes (comunidad universitaria) que se encuentran limitados por las estructuras que configuran dichos campos (Bourdieu, 2008) y que, en su relación, producen tensiones y toma de decisiones que afectan de forma directa o indirecta el acontecer contextual donde se ven reflejados las diversas formas de actuar $\left(\right.$ hábitus $\left.^{8}\right)$. Estos hábitus son al mismo tiempo producto de la historia colectiva de la organización (Ballester \& Colom, 2012).

El marco general de la estructuración de la universidad en términos de sus funciones sustantivas, se encuentra dividido en tres categorías centrales, función de docencia, función de investigación, función de extensión y una cuarta categoría emergente que se ha venido identificando y, que tradicionalmente, no se ha planteado como función propia de la universidad, pero que transversaliza y afecta de forma directa las otras tres funciones, llamada función de gestión interna administrativa. De esta forma, es posible afirmar que, desde las tres primeras funciones la universidad presenta una relación continua con la sociedad. Lo que permite entrever, que, es posible que existan unos impactos sociales y ambientales a partir de dicha relación.

Por otro lado, desde las relaciones intrainstitucionales, las funciones ejercen un riesgo social y ambiental a partir de las decisiones y actuaciones que se presentan en su interior. Según Franco (2018), un riesgo, que se asume como socioambiental, se puede concebir como el sentido de posibilidad (probabilidad indeterminada) que cada selección o decisión de la universidad como organización puede presentar al poner en acción sus funciones sustantivas, desde las cuales genera impactos en múltiples direcciones; pero que, según el mismo autor, se convierten en el sentido de actualidad (probabilidad determinada) de la decisión o alternativa seleccionada.

Teniendo presente lo planteado, es posible afirmar que, desde cada una de las funciones sustantivas de la universidad se van gestando riesgos (posibilidades) e impactos (actualidades) que afectan el acontecer social y ambiental en el cual interviene la universidad y que obligan a esta a plantear una perspectiva de gestión centrada en la responsabilidad social y en la responsabilidad ambiental.

Lo anterior, le puede servir a la universidad para construir e implementar prácticas de autoobservación y autoevaluación de sus procesos, $\mathrm{y}$, por consiguiente, a establecer condiciones que minimicen el impacto negativo que genera su actuar en la comunidad y el territorio, a partir de una profunda reflexión sobre los habitus, con el claro objetivo de generar comportamientos más sostenibles con el ambiente.

8 Bourdieu (2008) lo define como disposiciones, formas de obrar, sentir o pensar frente a posiciones netamente sociales. 


\section{Análisis de caso: manejo de residuos sólidos en la Universidad Católica de Oriente}

La Universidad Católica de Oriente (UCO) entiende como institución que tiene una función formativa, científica y de proyección social, y al mismo tiempo, como un escenario para la construcción de conocimiento. Por ello, propende por la divulgación y apropiación social del conocimiento, de forma tal que pueda llegar a transformar prácticas sociales, culturales y productivas a través de la implementación de conocimientos sociales, científicos y tecnológicos. En este sentido, la UCO preocupada por la relación con su entorno y por su posible impacto en el territorio, busca desarrollar nuevas formas de interactuar en lo educativo, científico, cultural, económico, social y ambiental.

En este contexto, la UCO desde su creación ha tomado como parte de su plataforma teleológica la RSA, como una forma de responder a las demandas del territorio que le ha dado su origen, con lo cual propende por cumplir objetivos concretos, estableciendo relaciones con la comunidad y con las instituciones con las que interactúa. Esta posición de responsabilidad la ha llevado a reflexionar en torno al acontecer de sus funciones sustantivas, haciendo suyos los temas respecto a la triada universidad-sociedad-ambiente, y reconociendo las dimensiones de riesgo e impacto que en ella se pueda presentar (Franco, 2018). Lo anterior corresponde con la categoría base social-contextual, derivada de la perspectiva latinoamericana de la RAU que refiere a la gestión del impacto de las funciones sustantivas sobre el contexto social-ambiental en el que se desenvuelve una organización.

Profundizando en el análisis de las prácticas de gestión y educación ambiental, es preciso destacar que la UCO inició en el año 2000 la implementación del sistema de gestión de la calidad bajo el modelo de las normas ISO, como un enfoque orientado al mejoramiento de la calidad de los servicios y al cumplimiento de los objetivos institucionales, la satisfacción de los usuarios internos y externos, y la identificación-aplicación de acciones de mejora continuas.

Con la consciencia de que el medio ambiente no puede considerarse como un aspecto apartado del accionar universitario, sino que se configura como una variable más del mismo, se definió la política ambiental en la institución, la cual tiene por fundamentos la misión institucional y el compromiso con el desarrollo sostenible (Acuerdo Consejo Directivo CD-020 de 2002) y la cual se materializó con la implementación del Sistema de Gestión Ambiental (SGA), igualmente bajo la metodología de la norma ISO 14001. De esta manera, se han generado espacios de discusión frente al fenómeno ambiental, riesgos e impactos de las actividades misionales, y se han invertido recursos en procura de consolidar una cultura ambiental entre los integrantes de la comunidad académica que trascienda su cotidianidad e impacte positivamente, tanto dentro del campus universitario como fuera de él. Asimismo, el SGA proporciona un indicador tanto del rendimiento o desempeño como del cum- 
plimiento normativo, lo cual ayuda a aumentar el nivel de confianza de los usuarios y del público en general, producto de la mayor transparencia en la gestión ambiental de la institución.

Ahora bien, se reconoce que el sistema sustentando en las ISO se inserta en las lógicas de estandarización y normalización de prácticas y procesos propios del mercado globalizado, y por ende, se asumen como una derivación de las teorías de la RSE expuesta anteriormente. En concreto, las referentes a la teoría institucional que se basa en los mecanismos de coerción ejercida a través de marcos normativos, el mimetismo (imitación de prácticas) y la estandarización (normalización) para lograr los cometidos de responsabilidad social.

De ahí que el solo modelo ISO resulte insuficiente en cuanto al logro de las intencionalidades educativas propias de una institución de esta naturaleza, específicamente en lo referente a lo ambiental y en cuanto a la incidencia del SGA sobre los currículos toda vez que dicho modelo se reduce al cumplimiento de los requerimientos contenidos en la norma (ISO). De facto, los esfuerzos se orientan más a lograr el cumplimiento del estándar internacional, que a conseguir la trazabilidad del impacto del sistema en las funciones sustantivas.

Como consecuencia de lo anterior, se han evidenciado en la UCO avances hacia la configuración de un SGA que incorpora elementos del concepto de responsabilidad ambiental universitaria surgido de la perspectiva latinoamericana aludida anteriormente, ello es, la concepción de una responsabilidad en términos sociales y ambientales frente a la comunidad educativa y frente al territorio.

En tal sentido, se identifican dos elementos asociados a la RAU en la UCO. Por un lado, el modelo fundamentado en las normas ISO (14001) del cual se destaca la documentación de procesos y procedimientos, la elaboración de la matriz de valoración de aspecto-impacto, las auditorías internas y externas, los informes de la dirección, entre otros, las cuales se convierten en referentes concretos, ya que están atadas a condicionales preceptivas en términos de la norma, como también en términos de política institucional, que a su vez restringen las valoraciones subjetivas.

Por otra parte, se evidencian apuestas por trascender el alcance de las ISO con estrategias tales como campañas educativas que apuntan al cuidado del ambiente, pero también a la formación de sujetos ecológicos y la definición dentro de la planeación estratégica de la Universidad (Plan de Desarrollo 2017-2022) del programa denominado Promoción de la Ciudadanía Ecológica, cuyo objetivo es:

Integrar la gestión ambiental a los procesos a partir de la responsabilidad del cuidado de la casa común, por medio del establecimiento de nuevas estrategias con las cuales la comunidad universitaria toma conciencia de la responsabilidad que tenemos con la creación en su entorno ambiental, social, político y económico, iluminados y guiados por la encíclica Laudato si' (UCO, 2017, p. 74). 
Como puede colegirse, el SGA se fortalece no solo con las perspectivas de la RSU sino también con postulados del Magisterio de la Iglesia que la institución acoge debido a su carácter confesional.

De lo anterior se deriva el subprograma denominado Fortalecimiento de la cultura ecológica a partir del cual la Universidad comprende "el desafío urgente de proteger nuestra casa común", [el cual] incluye la preocupación de unir a toda la familia humana en la búsqueda de un desarrollo sostenible e integral" (Francisco, 2015, citado en UCO 2017, p. 75). Así, las acciones operativas asociadas a este programa del plan estratégico institucional son:

- Diseñar e implementar estrategias pedagógicas orientadas a la consolidación de la cultura ecológica.

- Promover la cultura ecológica a través de la formación humanística impartida en la institución.

- Diseñar programas de servicio a la comunidad de monitoreo e intervención sobre el cuidado del medio ambiente a través de la articulación con las unidades que en la Universidad tienen esta responsabilidad (UCO, 2017, p. 75).

Ahora bien, en términos prácticos y acorde con lo expuesto hasta este punto, el SGA de la UCO tiene un componente de identificación de los aspectos que lo integran, los riesgos y la valoración de su impacto, cuyo resultado es su significancia técnica ambiental, determinada a través de la ponderación de los siguientes atributos: severidad, frecuencia, relevancia y control. Se trata de una matriz en la que se identifican los riesgos ambientales y se valoran de acuerdo con los atributos mencionados, permitiendo actuar sobre aquellos riesgos con un posible mayor impacto mediante la definición de los programas de intervención y actuación en materia ambiental ${ }^{10}$.

Si se analiza desde la perspectiva latinoamericana de la RAU, este ejercicio se correspondería con la categoría base objetiva-material según la cual se identifican los riesgos derivados de las decisiones relacionadas con los ambientes físicos en los cuales se desarrollan cada una de las funciones sustantivas. Por ejemplo, algunos de los principales riesgos que se han identificado y del cual han surgido programas ambientales, son: manejo seguro de residuos químicos y manejo integral de residuos sólidos en la fuente, orden y aseo, y uso eficiente y racional de los recursos naturales (energías y agua específicamente).

De hecho, el aspecto que ha presentado mayor complejidad es el concerniente al manejo de residuos sólidos toda vez que no se ha logrado generar la conciencia suficiente dentro de la comunidad educativa, encontrando resistencia en quienes habitan y frecuentan el campus universitario sin lograr asegurar una disposición responsa-

9 Es el término utilizado por el Papa Francisco en la Encíclica Laudato si' para referirse al planeta que habitamos.

10 Matriz de aspecto impacto (Universidad Católica de Oriente, 2007). 
ble de los residuos sólidos en la fuente. A esto se suma el hecho de que la norma internacional que define los colores de los recipientes en los cuales se debe hacer la separación y disposición de residuos, ha sido modificada constantemente, generando confusiones en la comunidad. A pesar de estas dificultades, SICE (2017) define algunas prácticas en el marco del sistema de manejo integral de residuos ${ }^{11}$, a saber:

- Gestión interna de residuos

- Gestión externa del residuo

- Identificación de puntos ecológicos y campañas de sensibilización

- Contabilización de residuos por área

- Caracterización de residuos

- Puntos ecológicos para la separación del papel

- Plan de medios

- Campañas de sensibilización ${ }^{12}$

Ahora bien, frente al manejo integral de residuos se han emprendido prácticas ambientales (como el reciclaje) y acciones ambientales (derivadas de estas como la separación de residuos) tendientes a identificarlos y separarlos desde la fuente, estableciendo una clasificación según la cual estos residuos pueden ser aprovechables o no aprovechables (figuras 2 y 3), fijando para cada tipo de acciones metas anuales de recolección, gestión y disposición.

Figura 2. Generación de residuos aprovechables 2010 - 2017

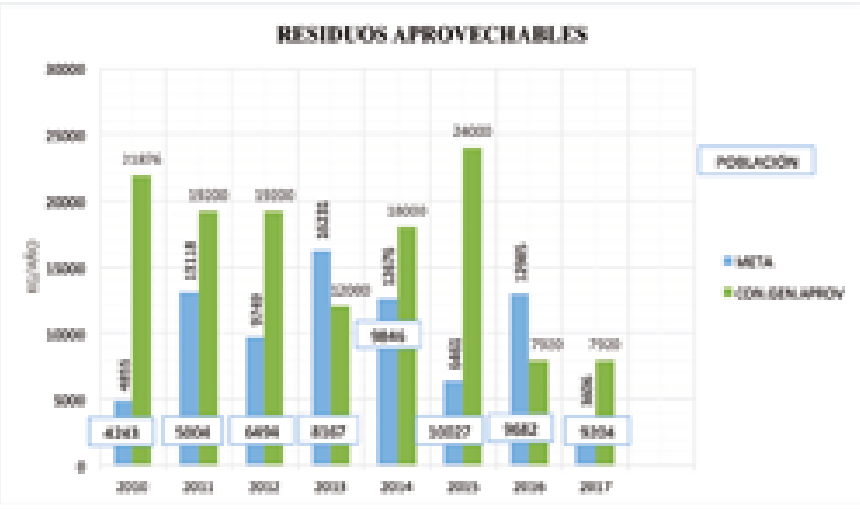

Fuente. Tomado de SICE (2017).

11 Para la presente investigación la caracterización y justificación de cada componente no tiene lugar, sin embargo, para aquel que quiere conocer en detalle, se sugiere ver SICE (2017). Plan de apoyo y mejoramiento del PMIRS de la Universidad Católica del Oriente, en el cual se describe la lógica y el proceso de forma detallada.

12 Campañas que se comparten con toda la comunidad universitaria, tales como recolección de la chatarra electrónica, llena una botella de amor, recolección de pilas, recolecciones de medicamentos usados y vencidos, entre otros. 
La figura 2 da cuenta de la generación de residuos aprovechables entre 2010 y 2017, y presenta una tendencia a la generación de este tipo de residuos por encima de las metas trazadas por la universidad, exceptuando los años 2013 y 2016. Dicha tendencia obedeció al incremento en el número de estudiantes y a la apertura de nuevos puntos de venta en el interior de la universidad. En tal sentido, se puede señalar que sobrepasar la meta ha generado un reto institucional que se acerca a una perspectiva de RAU derivada-de la RSE. Desde esta perspectiva es posible afirmar que se hace necesario el fortalecimiento de los procesos de acompañamiento a grupos de interés stakeholders como son los concesionarios de las cafeterías, organizaciones, operadores logísticos y la comunidad en general que generan a partir del eje organizacional impactos en términos laborales y ambientales externos e internos como se indica en la figura 1. Con todo, se resalta que este tipo de acciones ha permitido que estos residuos se traduzcan en ingresos económicos para la institución como producto de su venta al sector industrial que los reutiliza en sus procesos productivos, y que, aunque no representan un flujo importante de recursos financieros, sí contribuye a una mejor disposición de los residuos.

Figura 3. Generación de residuos no aprovechables periodo 2010-2017

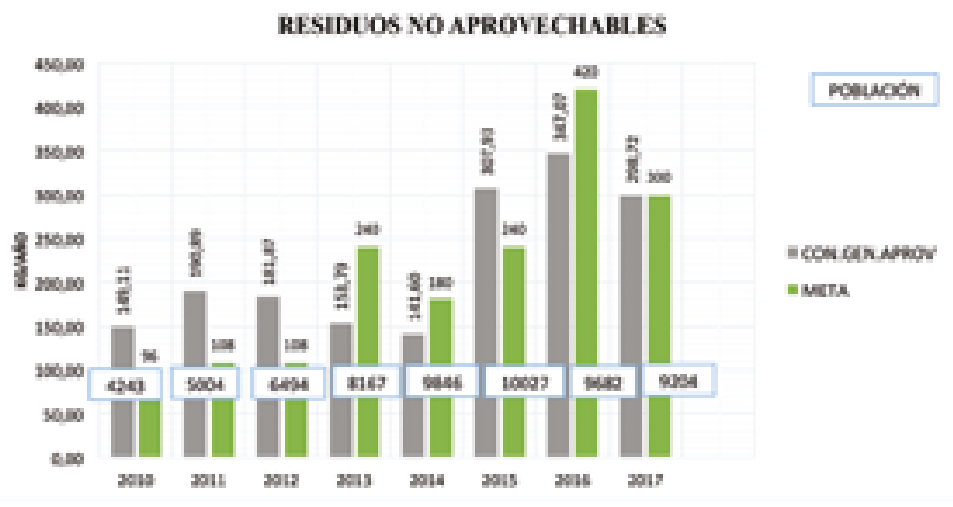

Fuente. Tomado de SICE (2017).

Por su parte, la figura 3 presenta el comportamiento de los residuos no aprovechables en el mismo período de tiempo, los cuales han tenido una tendencia creciente en el período 2015-2017 como consecuencia de la apertura de nuevos puntos de venta de comestibles dentro del campus y del crecimiento en el número de estudiantes matriculados en la institución. Como se evidencia en las figuras 2 y 3 el 2015 fue un año de inflexión en el que aumentaron los estudiantes y se elevó la generación de residuos, tanto aprovechables como no aprovechables. Sin embargo, en los dos años siguientes se identifica un incremento en los residuos no aprovechables y un decremento en los aprovechables que llevó a la generación de una acción de intervención con miras a atenuar este efecto, mediante la formulación y ejecución de un proyecto de compos- 
taje por aire forzado que permite hacer el tratamiento de este tipo de residuos y del cual se han presentado resultados favorables.

Por otro lado, también se han intervenido los residuos generados por la utilización de sustancias químicas, principalmente en laboratorios, mediante un proceso investigativo liderado por el programa de Ingeniería Ambiental, a través del cual se busca desactivar tales sustancias para evitar disponerlas en el relleno sanitario. También es de destacar las campañas posconsumo realizadas con la comunidad universitaria, las cuales están orientadas a asegurar la adecuada disposición de otros residuos que se generan, tanto en el interior del campus como en las residencias de los integrantes de la comunidad, como se evidencia en la figura 4.

Figura 4: Generación de residuos industriales período 2011-2016 y generación de residuos biológicos período 2010-2017

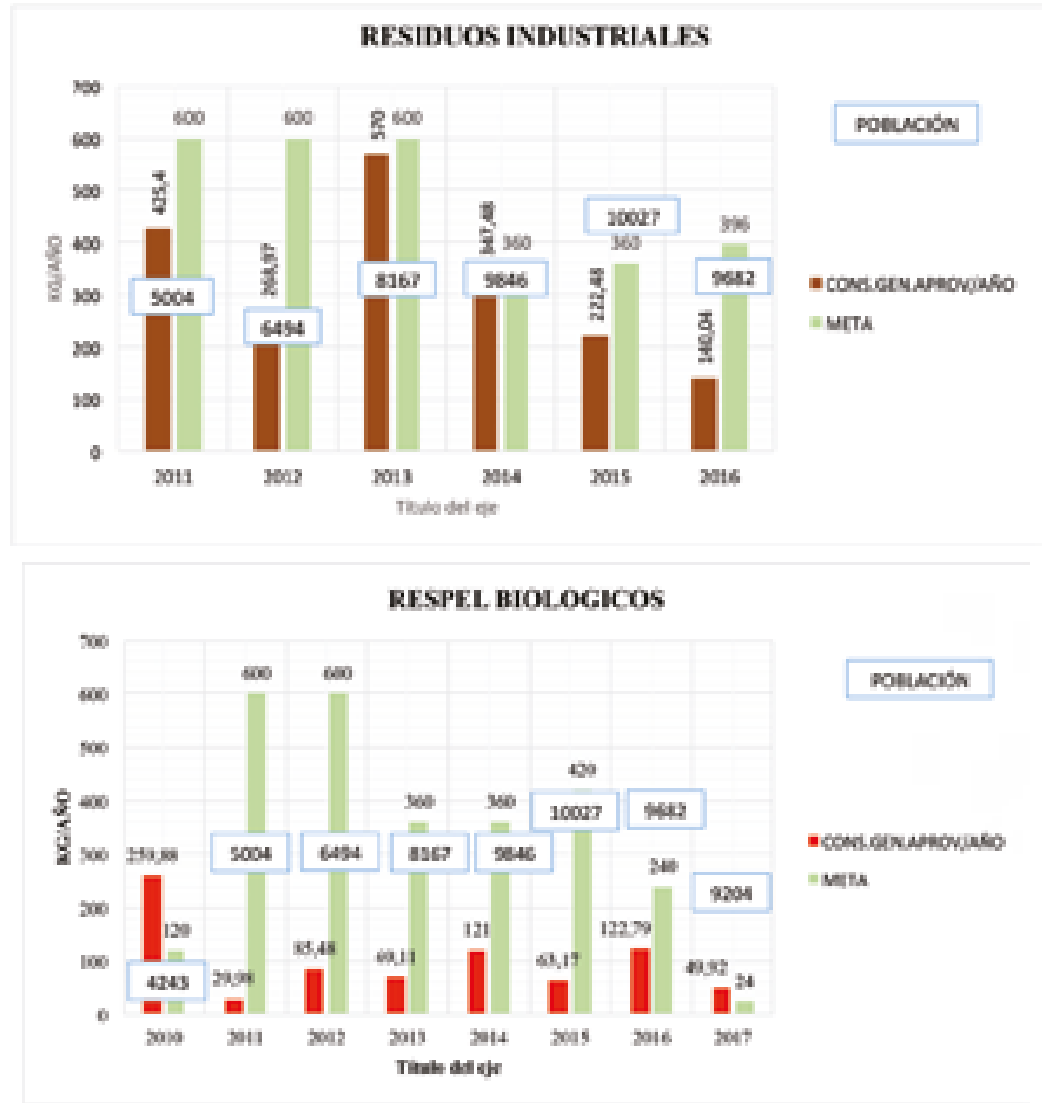

Fuente. Tomado de SICE (2017). 
La figura 4 muestra los residuos pertenecientes a la denominación RESPEL ${ }^{13}$, compuesto por residuos industriales y residuos biológicos, la cual evidencia una disminución en ambos tipos de residuos gracias a las prácticas ambientales de formación que a partir del eje académico (figura 1) generan impactos cognitivos y educativos en el personal a cargo de los laboratorios, docentes, estudiantes y servicios generales, que ha conllevado una mayor sensibilización frente a este tipo de residuos y, consecuentemente, a las buenas acciones ambientales implementadas por estos empleados.

En lo que respecta a la generación de residuos industriales, se evidencian niveles altos entre los años 2011 y 2013, lo cual obedece al cambio de lámparas en la institución para así mejorar el consumo de energía.

Así mismo, en el marco de esta práctica ambiental formativa se ha llevado la implementación de acciones, como: la ubicación de contenedores en el campus universitario en los que se recolectan pilas, chatarra electrónica y medicamentos usados o vencidos, los cuales se entregan a un "Gestor Responsable ${ }^{14}$ " encargado de la recuperación y disposición de manera responsable, tal y como se resume en la tabla 1, en la que se presenta la cantidad de residuos RESPEL, recolectados y dispuestos.

Tabla 2. Registro de recuperación y disposición de residuos RESPEL

\begin{tabular}{llll}
\hline Año & \multicolumn{1}{c}{ Medicamentos } & \multicolumn{1}{c}{ Chatarra electrónica } & \multicolumn{1}{c}{ Pilas } \\
\hline 2015 & $* * *$ & $126 \mathrm{~kg}$ & $* * *$ \\
2016 & $3 \mathrm{~kg}$ & $708 \mathrm{~kg}$ & $56 \mathrm{~kg}$ \\
2017 & $23 \mathrm{~kg}$ & $746 \mathrm{~kg}$ & $41.9 \mathrm{~kg}$ \\
\hline
\end{tabular}

Fuente. Tomado de SICE (2017).

De igual manera, existe otra acción ambiental denominada "llena una botella de amor", cuyo propósito es generar conciencia en la comunidad universitaria para la adecuada disposición de todo tipo de plástico, la cual ha permitido hacer una recolección de 577 kilogramos de plástico en total al cierre 2017. Cabe resaltar que la primera entrega fue de 201 kilogramos y permitió la construcción de cinco puntos ecológicos, cinco mesas de comedor y diez archivadores que beneficiaron a la Escuela Normandía del municipio de El Retiro. Esta acción puede ser leída en perspectiva de la RAU en clave de la RSE bajo el enfoque de legitimación que pretende la aceptación y aprobación de las diversas acciones ambientales (de extensión) emprendidas por la universidad y que repercuten en la percepción de las comunidades sobre esta por su connotación socio-contextual.

Por último, conviene hacer mención que las auditorías y otras acciones han permitido hacer mejoras al SGA en procura de su consolidación. Se resaltan en la si-

13 Registro de generadores de residuos o desechos peligrosos (Universidad Católica de Oriente, 2005).

14 Empleado de la Universidad Católica de Oriente encargado de la recuperación y disposición de manera responsable. 
guiente tabla las acciones ambientales de mejora identificadas y documentadas por el Comité de Gestión Ambiental.

Tabla 3. Relación entre acciones ambientales propuestas en la universidad y categorías en las que se inscribe

\begin{tabular}{ll}
\hline \multicolumn{1}{c}{ ACCIÓN AMBIENTAL } & CATEGORÍA EN LA QUE SE INSCRIBE \\
\hline $\begin{array}{l}\text { Participación en el proyecto Pacto Mundial de } \\
\text { Naciones Unidas (Global Compact). }\end{array}$ & RSE Institucional: mimética. \\
$\begin{array}{l}\text { La participación en la prueba piloto "Punto Limpio" } \\
\text { con el municipio de Rionegro, que consiste en un lugar } \\
\text { de recolección de residuos, que generan los vecinos de } \\
\text { la Universidad. }\end{array}$ & RSE institucional: de percepción. \\
\hline $\begin{array}{l}\text { El seguimiento a los expedientes CORNARE en } \\
\text { cumplimiento de las directrices definidas desde la }\end{array}$ & RSE institucional: de coerción/normalización/ \\
$\begin{array}{l}\text { Estrategia de Gobierno en Línea y la implementación } \\
\text { de la Ventanilla Integral de Trámites Ambientales ante } \\
\text { el ente territorial CORNARE. }\end{array}$ & mimesis \\
$\begin{array}{l}\text { Implementación de indicadores GRI, el cual da cuenta } \\
\text { de la elaboración de una memoria de sostenibilidad que } \\
\text { comprende la medición, divulgación y rendición de } \\
\text { cuentas frente a grupos de interés internos y externos } \\
\text { en relación con el desempeño de la organización con } \\
\text { respecto al objetivo del desarrollo sostenible. }\end{array}$ & \\
\hline
\end{tabular}

Fuente. Elaboración propia.

\section{Conclusiones}

Lo anterior denota que las prácticas de responsabilidad social, indiferente de su connotación (empresarial, universitaria o ambiental) se convierten en una oportunidad para las organizaciones, siempre y cuando los criterios de su implementación se encuentren alineados con los objetivos organizacionales, los cuales, dependiendo de los intereses que se estén persiguiendo puede ser económicos, políticos, éticos, o filantrópico (Carroll, 1991).

En esta oportunidad, el manuscrito sugiere que la universidad como administradora de un bien público (el conocimiento) sea quien determine o dé lineamientos claros a otras variables que se encuentran inmersas en las dinámicas organizacionales, pero que en algunos casos carecen de urgencia (Mitchell et al, 1997), como en el caso de la responsabilidad ambiental, la cual se ha convertido en una variable crítica para la administración actual.

También es preciso resaltar, que gestionar prácticas de responsabilidad social en realidades diferentes a las del sector privado, o para ser más claros a las realidades conocidas como "con ánimo de lucro", resulta en ejercicios con un alto nivel de subjetividad en cuanto a la rendición de cuentas, ya que el debate no descansa sobre parámetros concretos y se continúa avanzando sobre directrices o principios filosóficos 
y con un alto nivel filantrópico. Aunque habría que decir que el implementar estas prácticas mejora el vínculo emocional de los stakeholders con la universidad, lo que en el mediano o largo plazo mejora la competitividad de la misma.

Otro rasgo derivado, es el compromiso de la universidad por hacer una adecuada gestión de los riesgos e impactos ambientales generados por el desarrollo de sus funciones sustantivas, ampliando de alguna manera el alcance de la norma ISO 14001. Seguramente hará falta conceptualizar aún más sobre este asunto a efectos de hacerlo consciente y fortalecer teórica y conceptualmente el SGA para lo cual tendría bastante significado la perspectiva latinoamericana de la RAU y de la cual se tienen aproximaciones prácticas en dicho SGA universitario.

En efecto, podría afirmarse que las prácticas y acciones ambientales enunciadas anteriormente se plantean a partir de los riesgos identificados, pero tendrían como base las categorías denominadas: eje organización y eje académico, desde los cuales se pretende modificar la cultura ambiental respecto de cómo piensan, sienten, actúan y se interrelacionan los miembros de la institución.

De esta manera, la gestión ambiental bajo perspectivas empresariales, y en concreto la referida a la normalización de procesos bajo cualquiera de los estándares definidos para el efecto, resulta insuficiente en una institución educativa, puesto que dada su naturaleza formativa no debe reducir el SGA a una mirada instrumental y técnica, sino que por el contrario, debe hacer trascender el alcance y aplicación de la norma para alcanzar una auténtica dimensión de responsabilidad ambiental con impactos internos y externos. Para ello se hace necesario permear los diseños curriculares en todos los niveles de formación y las diferentes actividades que se adelanten en desarrollo de las funciones sustantivas.

\section{Referencias}

Ángel, N. (2008). La discusión en torno a las soluciones de soft law en materia de responsabilidad social empresarial. Revista de Derecho Privado, 40, 3-37.

Ashworth, R., Boyne, G., \& Delbridge, R. (2007). Escape from the iron cage? Organizational change and isomorphic pressures in the public sector. Journal of public administration research and theory, 19(1), 165-187.

Ballester, L., \& Colom, A. (2012). Epistemología de las ciencias sociales y de la educación. Tirant Humanidades.

Bohren, O. (1998). The Agent's Ethics in the Principal-Agent Model. Journal of Business Ethics, 17(7), 745-755.

Bourdieu, P. (2008). El sentido práctico. Siglo XXI de España Editores. 
Bustos, C., y Inciarte, A. (2012). (2012). Dimensión comunitaria de la responsabilidad social universitaria. Opción: 28(68), 367-379.

Camacho, I., Fernández, J., Miralles, J. (2005). Ética de la empresa. España: Univ. Jesuitas.

Carroll, A. B. (1991). The pyramid of corporate social responsibility: Toward the moral management of organizational stakeholders. Business Horizons, 34(4), 3948.

Cortese, A. D. (2003). The critical role of higher education in creating a sustainable future. Planning for Higher Education, 31(3), 15-22.

De la Torre, I. (2009). Fundamentos de la responsabilidad de la empresa. Revista de Responsabilidad Social de la Empresa (1), 71-89.

Evan, W., \& Freeman, R. (1988). A stakeholder theory of the modern corporation: Kantian capitalism. Ethical theory and business, 75-84.

Fassin, Y., Gosselin, D. (2011). The collapse of european bank in the financial crisis: an analysis from stakeholder and ethical perspectives. Journal of Business Ethics, 102(2), 169-191, doi: 10.1007/s10551-011-0812-2.

Franco, J. (2018). La responsabilidad social en la educación superior: una mirada integral desde la concepción de decisión, riesgo e impacto. En D. Pérez, D. Vélez, A. López, \& P. Múnera (Eds.). Responsabilidad social: Lecturas y debates. Rionegro, Colombia: Fondo Editorial Universidad Católica de Oriente.

Freeman, R. (1984). Strategic Management: A Stakeholder Approach. Boston: Pitman Publishing.

Gaete, R. (2015). The Concept of University Social Responsibility from the Perspective of Upper Direction. Cuadernos de Administración, 31(53), 97-107. Universidad del Valle. doi: 10.25100/cdea.v31i53.20.

Galán, J. I. (2006). Diseño organizativo. Madrid: Thomson.

Grant, R. (2006). Dirección estratégica: campos, técnicas y aplicaciones. Madrid: Civitas.

Gray, R., Owen, D., \& Adams, C. (1996). Accounting \& accountability: changes and challenges in corporate social and environmental reporting. Prentice Hall.

Jensen, M. C., \& Meckling, W. H. (1976). Theory of the firm: Managerial behavior, agency costs and ownership structure. Journal of Financial Economics, 3(4), 305-360.

Jiménez, A., Pérez, F. (2017). Universidad y territorio. Las misiones: una estrategia de extensión universitaria con sentido territoriall. Kénosis. Revista de Ciencias Sociales y Humanas, 5(8), 118-143. 
Jones, T. (1995). Instrumental Stakeholder Theory. The Academy of Management Review, 20(2), 404-437.

Lapsley, I. (1999). Accounting and the new public management: instruments of substantive efficiency or a rationalising modernity? Financial Accountability \& Management, 15(3-4), 201-207.

López, A. (2018). Más que un prólogo o una introducción, una invitación. En D. Pérez, D. Vélez, A. López, \& P. Múnera. (Eds.). Responsabilidad social: Lecturas y debates, (pp. 11-13). Rionegro, Colombia: Fondo Editorial Universidad Católica de Oriente.

Melle, M. (2007). La responsabilidad social dentro del sector público. Ekonomiaz: Revista Vasca de Economía, (65), 84-107.

Mitchell, R. K., Agle, B. R., \& Wood, D. J. (1997). Toward a theory of stakeholder identification and salience: Defining the principle of who and what really counts. Academy of Management Review, 22(4), 853-886. doi: 10.5465/ amr.1997.9711022105.

Murths, T., \& Lenway, S. (1998). Country capabilities and the strategic state: How national political institutions affect multinational corporations' strategies. Strategic Management Journal, 15(5), 113-119.

Ojeda, J., Álvarez, D. (2015). Responsabilidad social en las universidades: antecedentes, trayectorias y perspectivas. COEPES (12). Recuperado de http:// www.revistacoepesgto.mx/revistacoepes $12 /$ responsabilidad-social-en-lasuniversidades-antecedentes-trayectorias-y-perspectivas

Porter, M. E., \& Kramer, M. R. (2011, febrero). La creación de valor compartido. Harvard Business Review América Latina, 1-18.

Powell, W., \& Di Maggio, P. (1999). El nuevo institucionalismo en el análisis organizacional (comp.). México D.F.: Fondo de Cultura Económico.

Rasche, A., \& Esser, D. (2006). From Stakeholder Management to Stakeholder Accountability. Journal of Business Ethics, 65(3), 251-267. doi:10.1007/s10551005-5355.

Reverte, C. (2009). Determinants of corporate social responsibility disclosure ratings by Spanish listed firms. Journal of Business Ethics, 88(2), 351-366. doi: 10.1007/ s10551-008-9968-9.

Ross, S. A. (1973). The economic theory of agency: the principal's problem. The American Economic Review, 63(2), 134-139.

Santos, J., Núñez, M., \& Alonso, I. (2007). Responsabilidad social en las universidades: necesidad de la elaboración de una memoria de sostenibilidad consensuada. Cuadernos Aragoneses de Economía, 17(1), 127-146. 
Scholl H.J. (2001). Applying Stakeholder Theory to E-government. In: Schmid B., Stanoevska-Slabeva K., Tschammer V. (Eds.). Towards the E-Society. IFIP International Federation for Information Processing, vol 74. Springer, Boston, MA SICE, (2017). Sistema Integrado de Calidad Educativa de la Universidad Católica de Oriente. Rionegro, Antioquia.

Suchman, M. C. (1995). Managing legitimacy: Strategic and institutional approaches. Academy of Management Review, 20(3), 571-610.

Sousa, J. M., Outtes, L. S., Pasa, C., \& Farache, F. (2010). Strategic corporate social responsibility management for competitive advantage. Brazilian Administration Review, 7(3), 294-309.

Trujillo, M., \& Vélez, R. (2006). Responsabilidad ambiental como estrategia para la perdurabilidad empresarial. Universidad \& Empresa, 5(10), 291-308.

Vaca,R.,Moreno,M.,Riquel,F.(2007).Análisis delaresponsabilidad social corporativa desde tres enfoques: stakeholders, capital intelectual y teoría institucional. En J.C. Ayala Calvo y Grupo de Investigación FEDRA. Conocimiento, innovación y emprendedores: camino al futuro. La Rioja: Universidad de la Rioja.

Vallaeys, F. (2014). La responsabilidad social universitaria: un nuevo modelo universitario contra la mercantilización. Revista Iberoamericana de Educación Superior, 5(12), 105-117.

Vilanova, M., Lozano, J., \& Arenas, D. (2009). Exploring the Nature of the Relationship Between CSR and Competitiveness. Journal of Business Ethics, 87(1), 57-69. doi: 10.1007/s10551-008-9812-2. 